\title{
PARASITOSES INTESTINAIS EM USUÁRIOS DO SUS EM LIMOEIRO DO NORTE, CEARÁ, ANTES DE EXPANSÃO DE SISTEMA DE ESGOTAMENTO SANITÁRIO
}

\author{
C. V. A. MAIA ${ }^{1 *}$, I. C. HASSUM ${ }^{2}$ e G. S. VALLADARES \\ ${ }^{1}$ Universidade Federal do Piauí - UFPI \\ ${ }^{2}$ Pesquisadora da Empresa Brasileira de Pesquisa Agropecuária - Embrapa \\ cvamaia@yahoo.com.br
}

Artigo submetido em fevereiro/2014 e aceito em abril/2015

DOI: 10.15628/holos.2015.1973

\section{RESUMO}

As parasitoses intestinais representam um agravo de amplitude mundial, sendo indicador de status socioeconômico. Sua transmissão feco-oral reforça a necessidade de ampliação de serviços de saneamento básico. $O$ estudo tem caráter retrospectivo, descritivo e exploratório, com dados secundários, objetiva apresentar a frequência parasitária em usuários do Sistema Único de Saúde (SUS), em Limoeiro do Norte CE, entre janeiro de 2008 e dezembro de 2009. Os exames foram realizados por meio da técnica de sedimentação espontânea de fezes (HPJ, 1934). Os dados foram organizados e analisados por meio de estatística descritiva, através dos testes de Quiquadrado, teste $t$ para amostras independentes, teste de Mann-Whitney e correlçao de Pearson, ambos com significância de $5 \%(p<0,05)$. Foram analisadas 1266 amostras, onde 295 (23,3\%) foram positivas para algum parasita. Endolimax nana, Entamoeba coli e Giardia lamblia foram os parasitas mais frequentes. O sexo masculino foi o mais acometido. Houve maior prevalência em adultos $(p<0,05)$. Verificou-se diferença estatística quanto à prevalência entre as Unidades Básicas de Saúde (UBSs) e entre a sazonalidade durante os anos $(p<0,05)$. A relação entre a prevalência e médias pluviométricas durante os meses apresentou correlação muito baixa $(r=-0,20)$ e sem significância estatística. Mesmo com a alta prevalência, não pode desconsiderar que a mesma esteja subestimada

PALAVRAS-CHAVE: parasitos, frequência, Sistema Único de Saúde, saneamento básico

\section{INTESTINAL PARASITES IN SUS USERS IN LIMOEIRO DO NORTE, CEARÁ BEFORE EXPANSION OF SEWAGE SYSTEM}

\begin{abstract}
The intestinal parasites represent a wrong global scale, being a indicator of socioeconomic status. His fecal-oral transmission reinforces the need for expansion of sanitation services. The study is retrospective, exploratory and descriptive, using secondary data, objectives to show the parasitic frequency in users of the Sistema Único de Saúde (SUS) in Limoeiro do Norte CE, between January 2008 and December 2009. The exams were conducted using the technique of spontaneous sedimentation of feces (HPJ, 1934). The data were organized and analyzed using descriptive statistics, through the chi-quare, independent sample $t$ test, Mann- Whitney test and Pearson correlation, with
\end{abstract}

$5 \%$ significance $(p<0.05)$. Were analyzed 1266 samples, where 295 (23.3\%) were positive for any parasite. Endolimax nana, Entamoeba coli and Giardia lamblia were the most common parasites. Men were more affected. There was higher prevalence in adults ( $p<$ 0.05 ). There was statistical difference in prevalence between the Basic Health Units (UBSs) and between seasonality over the years $(p<0.05)$. The relation between prevalence and average rainfall during the months showed very low correlation $(r=-0.20)$ and not statistically significant. Despite the high prevalence, can not discount possible underestimated prevalence.

KEYWORDS: parasites, frequency, Public Health System, sanitation 


\section{INTRODUÇÃO}

O enteroparasitisimo é o estado de infecção em que a agressão repercute prejudicialmente sobre o hospedeiro, causado mormente por protozoários e helmintos. Mais recorrente na África, Ásia e América Latina, ocorrendo em áreas urbanas, especialmente em regiões periféricas e favelas, e rurais, presente em todas as faixas etárias, constituindo indicador de status socioeconômico, de adequabilidade de serviços de saneamento, salubridade e de saúde pública de uma região (SANTOS; MERLINI, 2010; SILVA et al., 2010; FERNANDES et al., 2011 BELLIN; GRAZZIOTIN, 2011; LODO et al., 2010; SILVA et al. 2009; BELO et al., 2012).

Dentre seus sintomas, quando se manifestam, ocorrem diarreias, anemia, colites, desnutrição, interferência no desenvolvimento cognitivo e físico (jovens e crianças), alterações psicossociais e até óbitos. Suas consequências estão associadas à carga parasitária que o indivíduo carrega (CIMERMAN e CIMERMAN, 2008; FERREIRA et al., 2006; ADDUM et al., 2011; SANTOS; SANTOS; SOARES, 2007; RODRIGUES; CODEIRO; ATHAYDE, 2013).

A ocorrência deste parasitismo depende da idade, imunidade, alimentação, hábitos, tensão emocional do hospedeiro, constituição e contaminação do solo, índice de aglomerado populacional, condições sanitárias, ambientais, socioeconômicas, educacionais, presença de animais domésticos no peridomicílio, contaminação da água e dos alimentos, do potencial biótico e patogênico dos parasitas, o que favorecerá maior ou menor prejuízo ao hospedeiro (BELLIN; GRAZZIOTIN, 2010; COURA et al., 1994).

No Brasil, até os anos 70, realizaram-se grandes inquéritos coproparasitológicos. Atualmente, conta-se apenas com trabalhos pontuais em grupos específicos, que pela diversidade geográfica, social, econômica, sanitária, ambiental e cultural, nem sempre podem ser comparados. Mesmo assim, nota-se que em regiões com infraestrutura sanitária inadequada, alta é a prevalência do parasitismo, e que a gradativa melhoria dos serviços de saneamento proporcionam sua recrudescência (SOUZA; LUCCA; ANIBAL, 2006; MAGALHÃES; CARVALHO; FREITAS, 2011; CANTUARIA et al., 2011; FURTADO; MELO, 2010; OLIVEIRA, 2013; BERNADES et al., 2013; LUDUWING et al., 1999; BARRETO, 2007).

A comum transmissão feco-oral desta patologia reforça a necessidade de ampliações de serviços de saneamento básico e implementação de programas de educação sanitária e ambiental, por se caracterizarem como ações primárias de controle parasitológico.

A distribuição da infecção com diferenças inter e intrarregionais pode indicar condições desiguais de fatores socioeconômicos e de equipamentos sanitários, sendo, ocasionalmente, as populações hipossuficientes as que mais são acometidas, as que registram maior carga parasitária e as que possuem menos acesso a serviços públicos e equipamentos sociais, como, serviços de saneamento básico, sendo necessárias políticas públicas de cunho social e redistributivo (COSTA et al., 2012; SANTOS; SANTOS; SOARES, 2007).

É importante o conhecimento da prevalência enteroparasitária e sua análise descritiva em cada região, a fim de possibilitar o estabelecimento de medidas profiláticas e curativas, no intuito de minimizar a quantidade de indivíduos parasitados e fomentar subsídios para futuras políticas públicas (FONSECA et al., 2010). 
Este trabalho objetiva apresentar a frequência parasitária em usuários do Sistema Único de Saúde (SUS), em Limoeiro do Norte - CE, entre janeiro de 2008 e dezembro de 2009, período onde se insere o início da expansão do serviço de esgotamento sanitário local.

\section{METODOLOGIA}

\subsection{Caracterização da área}

Limoeiro do Norte (Figura 1), situado a $200 \mathrm{~km}$ da Capital, Fortaleza, inserida na microrregião do Baixo Jaguaribe, abrange a 25a maior população do Estado, com 56.281 habitantes, área de $751,535 \mathrm{~km}^{2}$ e densidade demográfica de 74,87 habitantes $/ \mathrm{km}^{2}$. Desses, 32.502 habitantes $(57,75 \%)$ residem no perímetro urbano, a Sede, habitando 11.058 domicílios $57,43 \%$ do total (IBGE,2010).

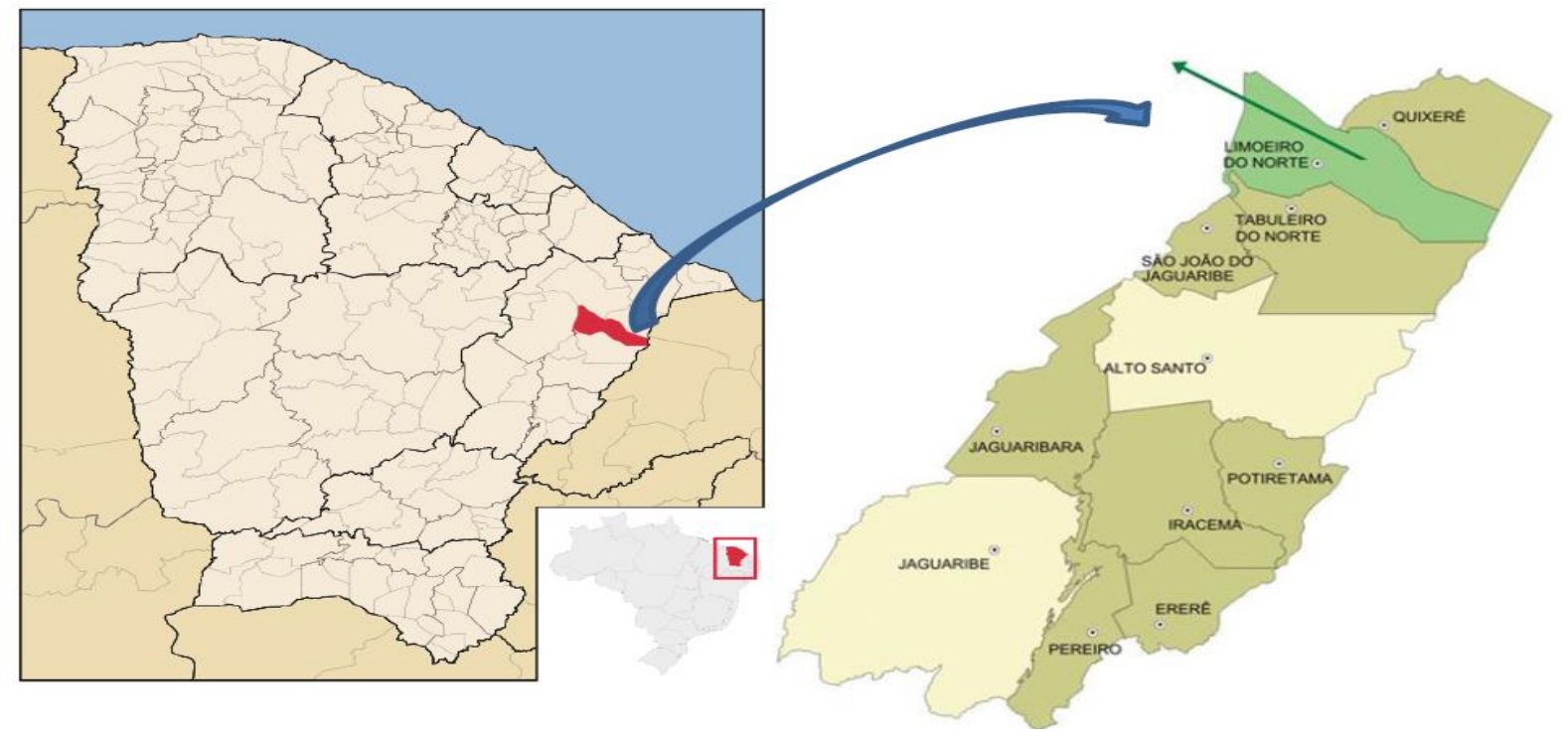

Figura 1-Localização do município de Limoeiro do Norte - CE.

A prestação dos serviços de saneamento, à época, se encontrava delegada a duas instituições públicas municipais. Drenagem urbana e gestão dos resíduos sólidos estavam a cargo da Secretaria Municipal de Infraestrutura e Desenvolvimento, enquanto que o Serviço de Abastecimento de Água (SAA) e Serviço de Esgotamento Sanitário (SES) eram gerenciados por uma autarquia municipal - Serviço Autônomo de Água e Esgoto (SAAE).

No período, os índices de cobertura do SAA e do SES, correspondiam a $86 \%$ e $11 \%$, respectivamente. O município dispunha de 13 Unidades Básicas de Saúde (UBSs), sete na Zona Rural e seis na Zona Urbana, porém, insuficientes, existindo, logo, áreas descobertas.

\subsection{Levantamento de dados}

O estudo foi do tipo ecológico, com abordagem retroativa, descritiva e exploratória, realizado no Laboratório do Hospital Regional Deoclécio Lima Verde (LHDLV), aonde os usuários do SUS procedem. Foram utilizados dados secundários obtidos juntos a Secretaria Municipal de Saúde, salvaguardando o anonimato dos pacientes. 
Foram analisados 1266 procedimentos coproscópicos, sob a técnica de sedimentação espontânea (HPJ, 1934) em usuários do SUS advindos das 13 UBSs municipais e de áreas descobertas, entre o período de janeiro de 2008 a dezembro de 2009.

Para fins de análise, foi considerado o complexo Entamoeba hystolistica/díspar, pelo fato de que a técnica utilizada, não permite distinção entre ambas.

De acordo com Rocha et al. (2010), as faixas etárias categorizadas pela Organização Mundial de Saúde em que são considerados indivíduos com idade entre 0 e 9 anos (crianças); entre 10 e 19 (adolescentes); de 20 a 59 (adultos) e com idade acima de 60 anos (idosos),foram consideradas neste levantamento.

Dados pluviométricos municipais foram coletados na página eletrônica da Fundação Cearense de Meteorologia e Recursos Hídricos- FUNCEME (2013), que disponibiliza série histórica em três postos de coletas no município, de onde foi feita média aritmética simples.

Os dados foram organizados e analisados por meio de estatística descritiva, através dos testes de Qui-quadrado, teste $\mathrm{t}$ para amostras independentes, teste de Mann-Whitney e correlçao de Pearson, ambos com significância de $5 \%(p<0,05)$.

\section{RESULTADOS E DISCUSSÕES}

Dos 1266 resultados coproparasitológicos verificados, 295 foram positivos para endoparasitos do trato digestório, correspondendo a uma taxa de prevalência de 23,3\%, dos quais $75,9 \%, 18,0 \%$ e $6,1 \%$ representaram mono, bi e poliparasitismo, respectivamente.

A prevalência encontrada é similar ao indicado pela Organização Mundial de Saúde (BELLIN; GRAZZIOTIN, 2011), que aponta a existência de um indivíduo parasitado, a cada quatro pessoas no mundo, e um pouco inferior ao que Ludwinget al. (1999) apresentaram em sua pesquisa que relaciona usuários de serviço público de saúde ao longo da implementação de obras de saneamento em Assis - SP, cuja prevalência inicial verificada foi de $28,5 \%$.

Basso et al. (2008), em Caxias do Sul, RS, encontrou prevalência maior, variando de $89 \%$ a $37 \%$.Os autores ainda verificaram que as melhorias sanitárias no decorrer de 35 anos induziram uma redução de $1,4 \%$ ao ano na prevalência.

Tabela 1 -Frequência enteroparasitária nos exames coprológicos de pacientes do SUS(2008-2009).

\begin{tabular}{c|c|c|c}
\hline GRUPO & GÊNERO/ESPÉCIE & OCORRÊNCIA(S) & FREQUÊNCIA (\%) \\
\hline Protozoário & Endolimax nana & 171 & 44,19 \\
\hline Protozoário & Entamoeba coli & 106 & 27,39 \\
\hline Protozoário & Giardialamblia & 63 & 16,28 \\
\hline Protozoário & Iodamoeba sp. & 24 & 6,2 \\
\hline Protozoário & Entamoebahistolystica/dispar & 17 & 4,4 \\
\hline Helminto & Enterobius vermiculares & 3 & 0,77 \\
\hline Helminto & Ancylostomasp. & 2 & 0,51 \\
\hline Helminto & Ascaris lumbricoides & 1 & 0,26 \\
\hline
\end{tabular}

Fonte - Hospital Regional Deoclécio Lima Verde, Limoeiro do Norte - CE

A maximização da carga parasitária pode estar associada à exposição mais frequente a fatores de risco ambiental, como: contato com resíduos de diversas naturezas e com água 
contaminada por fezes humanas, além de fatores de risco do ambiente familiar, tais como: condições de moradia (saneamento inadequado), práticas de higiene e hipossuficiência econômica (SILVA JUNIOR, 2010; FONTBONNE et al., 2011).

Foram encontrados 387 parasitos, dispostos em oito espécies, cinco do grupo dos protozoários e três compondo o grupo dos helmintos. Os protozoários de maior ocorrência foram Endolimax nana, Entamoeba coli e Giardia lamblia, conforme representa a Tabela 1.

Estes parasitas também foram os que tiveram maior frequência nas pesquisas de Bellin e Grazziotin (2011), Matos e Cruz (2012), Lodo et al. (2010), Santos et al. (2007), Menezes et al. (2013), todos envolvendo usuários do SUS.

A alta prevalência de protozoários (98,5\%) foi similar ao encontrado por Lodo et al. (2010) e por Matos e Cruz (2012) em usuários SUS em Bom Jesus dos Perdões, SP e Ibiassucê, BA, cujas taxas de presença de protozoários estavam acima de $93 \%$ em ambos os casos.

Verifica-se o caráter comensal que $E$. nana e $E$. coli assumem, por utilizarem o intestino como abrigo e fonte de alimento, porém, segundo Toledo (2009), o E. nana, quando em alta prevalência, associa-se a quadros diarreicos infantis (MENEZES et al., 2013).

A presença destes parasitos nos exames de fezes, geralmente, demonstra contaminação feco-oral, portanto, pode ser indicador de saneamento inadequado, ou mesmo inexistente, além de estarem associados à ocorrência de outros parasitas entéricos, como a Giardia lamblia, com prevalência de $16,3 \%$ neste estudo.

A Giardia lamblia pode ser transmitida diretamente pelo homem (aperto de mãos), ou indiretamente, através de ingestão de água contaminada, haja vista que este agente patológico é capaz de resistir à cloração da água, apresentando taxas interregionais variantes de prevalência no Brasil (ALMEIDA et al., 2010; REY, 2002).

Em Cumari, GO, a prevalência foi de 33,6\%, enquanto que em Goiorê, PR, foi de 13,5\%. Em Chapadinha, MA, foi identificada taxa de 25,4\%, no Macapá, AP, a frequência foi de $19,1 \%$ e 14,9\% em Bom Jesus dos Perdões, SP (BORGES; MACIANO; OLIVEIRA, 2010; OLIVEIRA; CHIUCHETTA, 2010; SILVA et al., 2010; MENEZES et al., 2013, LODO et al., 2010).

Foi observado que os homens foram os pacientes que mais buscaram o hospital para fazer exames copropológicos, correspondendo a $60,5 \%$ do total, representando $64,3 \%$ do parasitismo registrado, cuja distribuição de carga parasitária por gênero (Tabela 2) não representou diferença significativa pelo teste de Qui-quadrado $(p=0,16)$.

Tabela 2 - Carga parasitária por gênero em Limoeiro do Norte, CE.

\begin{tabular}{|c|c|c|c|c|c|c|c|c|c|}
\hline \multirow{3}{*}{ Sexo } & \multicolumn{8}{|c|}{ Carga } & \multirow{3}{*}{ Total } \\
\hline & \multicolumn{2}{|c|}{$\begin{array}{c}\text { Ausente } \\
p=0,13\end{array}$} & \multicolumn{2}{|c|}{$\begin{array}{c}\text { Monoparasitado } \\
\qquad p=0,71\end{array}$} & \multicolumn{2}{|c|}{$\begin{array}{c}\text { Biparasitado } \\
p=0,02^{*}\end{array}$} & \multicolumn{2}{|c|}{$\begin{array}{l}\text { Poliparasitado } \\
\qquad p=0,96\end{array}$} & \\
\hline & $\mathrm{N}$ & $\%$ & $\mathrm{~N}$ & $\%$ & $n$ & $\%$ & $\mathrm{n}$ & $\%$ & \\
\hline Feminino & 394 & 31,2 & 86 & 6,7 & 13 & 1,1 & 7 & 0,6 & 500 \\
\hline Masculino & 577 & 45,6 & 138 & 10,9 & 40 & 3,1 & 11 & 0,8 & 766 \\
\hline Total & 971 & 76,8 & 224 & 17,6 & 53 & 4,2 & 18 & 1,4 & 1266 \\
\hline
\end{tabular}

Fonte - Hospital Regional Deoclécio Lima Verde, Limoeiro do Norte - CE.

A diferença de positividade entre os sexos pode ser justificada pelos hábitos e labor, intrínsecos de cada gênero. $O$ sexo masculino foi quem mais realizou exames 
coproparasitológicos e apresentou maior proporção de biparasitismo, segundo o teste do Quiquadrado aos pares.

Em Aracaju (SE), Lopes et al. (2013) detectaram prevalência de 70,7\% em homens e justifica o fato pelo incremento fomentado pelo Ministério da Saúde, através do Programa de Política Nacional de Atenção Integral à Saúde do Homem, que almeja mudança de conduta quanto à saúde masculina.

Quanto à faixa etária, constatou-se, através do teste de Qui-quadrado, diferença significante $(p=0,03)$ quanto ao acometimento de indivíduos por faixa etária, sendo adolescentes e adultos os mais infectados, conforme Tabela 3.

Tabela 3 - Parasitismo por faixas etárias, Limoeiro do Norte, CE.

\begin{tabular}{c|c|c|c}
\hline Faixa Etária & Número de exames & Casos positivos/(\%) & $\%$ de casos positivos \\
\hline Criança & 358 & $59 /(16,5)$ & 20,1 \\
\hline Adolescente & 166 & $46 /(27,7)$ & 15,6 \\
\hline Adulto & 681 & $177 /(26,0)$ & 60,2 \\
\hline Idoso & 61 & $13 /(21,3)$ & 4,1 \\
\hline TOTAL & 1266 & $295 /(23,3)$ & 100 \\
\hline \multicolumn{4}{r}{ Fonte - Hospital Regional Deoclécio Lima Verde, Limoeiro do Norte - CE }
\end{tabular}

Neste período, a UBS com maior prevalência foi a denominada Serra, com 66 casos $(22,4 \%)$, localizada na zona rural, de onde foram confirmados, ao todo, 169 casos positivos (57,3\%), a qual se segue a zona urbana, responsável por 77 confirmações $(26,1 \%)$ e as Áreas Descobertas), demandantes de 49 casos positivos (16,6\%), conforme Tabela 4.

Tabela 4 - Frequência de indivíduos enteroparasitados diagnosticados em diferentes UBSs.

\begin{tabular}{|c|c|c|c|c|c|}
\hline UBS & ZONA & N Total & N Positivo & \% Positivo por UBS & $\begin{array}{l}\text { \% Positivo em } \\
\text { relação ao tota }\end{array}$ \\
\hline Serra & Rural & 159 & 66 & $41,5^{*}$ & 22,4 \\
\hline Várzea do Cobra & Rural & 199 & 34 & 17,1 & 11,5 \\
\hline Espinho & Rural & 153 & 24 & 15,7 & 8,1 \\
\hline Setores & Rural & 121 & 19 & 15,7 & 6,4 \\
\hline Córrego de Areia & Rural & 55 & 13 & 23,6 & 4,4 \\
\hline Bixopá & Rural & 47 & 10 & 21,3 & 3,4 \\
\hline Arraial & Rural & 30 & 3 & 10,0 & 1,1 \\
\hline \multicolumn{2}{|c|}{ Total na Zona Rural } & 764 & 169 & 22,1 & 57,3 \\
\hline Luís Alves & Urbana & 79 & 25 & 31,6 & 8,5 \\
\hline Centro & Urbana & 88 & 22 & 25,0 & 7,5 \\
\hline Boa Fé & Urbana & 53 & 13 & 24,5 & 4,4 \\
\hline Antônio Holanda & Urbano & 41 & 7 & 17,1 & 2,3 \\
\hline Centro II & Urbano & 41 & 7 & 17,1 & 2,3 \\
\hline José Simões & Urbano & 31 & 3 & 9,7 & 1,1 \\
\hline \multicolumn{2}{|c|}{ Total na Zona Urbana } & 333 & 77 & 23,1 & 26,1 \\
\hline \multicolumn{2}{|c|}{ Total em Áreas Descobertas } & 169 & 49 & 29,0 & 16,6 \\
\hline
\end{tabular}

Na Zona Urbana houve diferença significativa entre Luís Alves e José Simões $(p \leq 0,05)$. Houve diferença significativa entre a frequência por UBS $(p<0,01)$, sendo a UBS Serra a que apresenta maior prevalência, mas quando categorizadas por Região municipal (zona urbana, zona rural e áreas descobertas), não houve diferença significativa. 
Maior prevalência em comunidades rurais pode ser observada no trabalho realizado em Itambé do Mato Dentro (MG), com usuários do SUS, onde Mati et al., (2011) condicionam as diferenças ao acesso aos serviços de saneamento básico.

É possível que indivíduos residentes na zona urbana, com melhores condições de serviços de saneamento, estão passíveis a acometimento igual ou superior aos residentes em áreas rurais, pois existem outros fatores intervenientes, como: aglomeração populacional, consumo de alimentos infectados e sem a devida higienização (BORGES et al., 2011).

A sazonalidade do evento em Pelotas (RS) e em Bom Jesus dos Perdões (SP), que apresentam aspectos meteorológicos diferentes do Semiárido cearense, apontou maior prevalência no verão ou próximo a ele (entre dezembro e março), sob justificativa do pico de reprodução dos parasitas ocorrerem durante esta estação (DIAS, 2005; LODO et al., 2010).

As médias pluviométricas municipal, número de casos positivos e negativos e procedimentos realizados ao longo do biênio podem ser verificados na Tabela 5.

Tabela 5 - Distribuição temporal pluviométrica e de casos de enteroparasitoses.

\begin{tabular}{|c|c|c|c|c|}
\hline Mês & Média Pluviométrica $(\mathrm{mm})$ & Procedimentos & Casos Positivos & \% Casos positivos/mês \\
\hline Janeiro & 91,7 & 31 & 5 & 1,7 \\
\hline Fevereiro & 6,9 & 27 & 6 & 2,0 \\
\hline Março & 352,6 & 55 & 6 & 2,0 \\
\hline Abril & 195,4 & 57 & 12 & 4,0 \\
\hline Maio & 93,7 & 46 & 10 & 3.4 \\
\hline Junho & 11,5 & 43 & 8 & 2,7 \\
\hline Julho & 8,3 & 29 & 5 & 1,7 \\
\hline Agosto & 8,4 & 31 & 8 & 2,7 \\
\hline Setembro & 1,3 & 31 & 10 & 3,4 \\
\hline Outubro & 0,0 & 27 & 3 & 1,1 \\
\hline Novembro & 0,3 & 52 & 7 & 2,3 \\
\hline Dezembro & 0,0 & 28 & 3 & 1,1 \\
\hline Parcial 2008 & 770,3 & 457 & 83 & 28,1 \\
\hline Janeiro & 83,8 & 38 & 3 & 1,1 \\
\hline Fevereiro & 154,7 & 77 & 15 & 5,1 \\
\hline Março & 190,9 & 65 & 17 & 5,7 \\
\hline Abril & 437,7 & 26 & 3 & 1,1 \\
\hline Maio & 247,7 & 45 & 12 & 4,1 \\
\hline Junho & 30,9 & 50 & 14 & 4,7 \\
\hline Julho & 9,3 & 109 & 43 & 14,6 \\
\hline Agosto & 23,0 & 54 & 9 & 3,0 \\
\hline Setembro & 0,0 & 26 & 4 & 1,3 \\
\hline Outubro & 0,0 & 93 & 19 & 6,4 \\
\hline Novembro & 0,0 & 146 & 45 & 15,3 \\
\hline Dezembro & 9,1 & 80 & 28 & 9,5 \\
\hline Parcial 2009 & 1187,0 & 809 & 212 & 71,9 \\
\hline Total & 1957,3 & 1266 & 295 & 100 \\
\hline
\end{tabular}

Fonte: Hospital Regional Deoclécio Lima Verde, Limoeiro do Norte - CE e FUNCEME

$O$ teste $t$ apontou diferença estatística significativa entre as médias de casos positivos ( $p=$ $0,025)$ quando comparados os dois anos, com os últimos doze meses registrando maior índice de casos positivos $(71,9 \%)$. 
Não foi possível identificar diferença estatística significativa quanto à distribuição pluviométrica média pelo teste de Mann-Whitney $(p=0,487)$, mesmo que em 2009 tenha maior volume pluviométrico (1187 mm).

Os meses que registraram maiores índices de positividade estavam concentrados no último semestre de 2009, sendo, por ordem, novembro, julho e dezembro (Figura 2), diferindo das maiores precipitações que ocorreram entre fevereiro e junho nos dois anos, conforme pode ser observado na Figura 3.

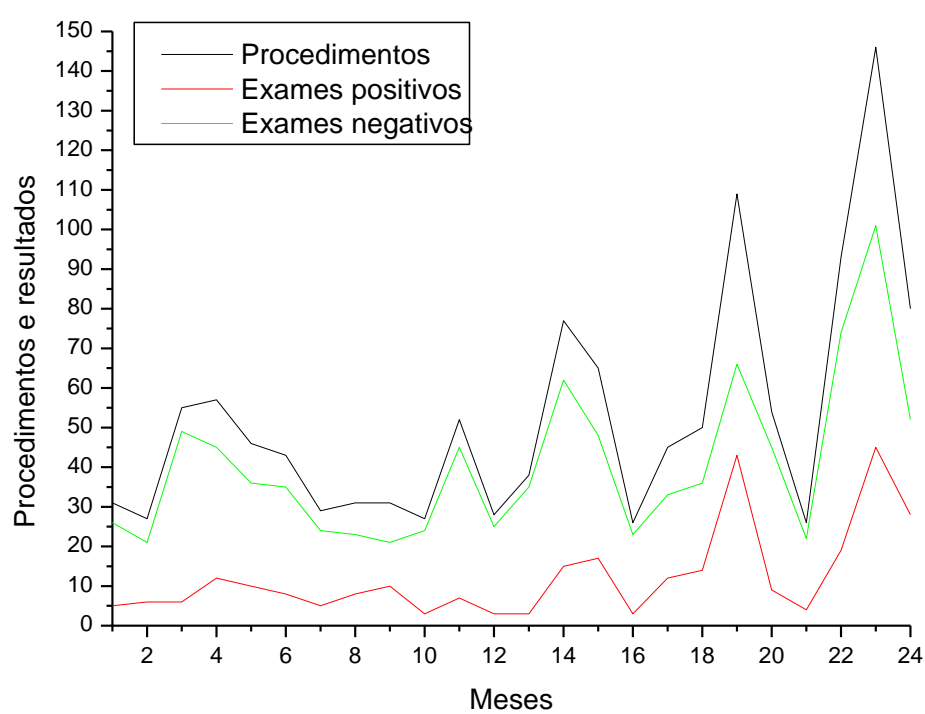

Figura 2 - Distribuição temporal dos casos de enteroparasitoses no HRLN

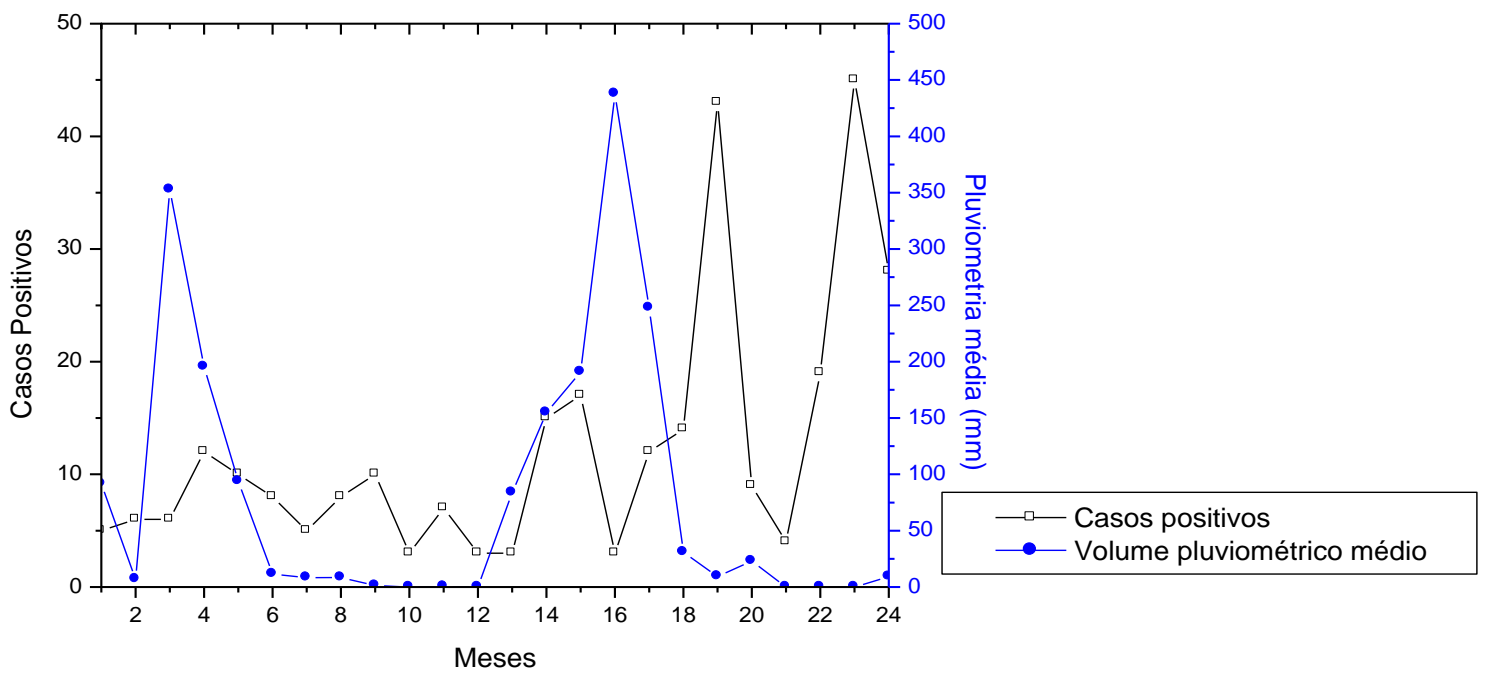

Figura 3 - Distribuição temporal dos casos positivos de enteroparasitoses e volume pluviométrico médio $(\mathrm{mm})$ em Limoeiro do Norte - CE

A correlação pelo teste de Pearson entre o número de casos positivos e os índices pluviométricos não foi siginificativa $(r=-0,20)$. Indicando não haver relação entre as duas variáveis. 
Salienta-se que mesmo com a alta prevalência encontrada, não se pode desconsiderar o fato de ela estar subestimada, haja vista que a metodologia empregada para o diagnóstico (sedimentação espontânea) utiliza somente uma lâmina, desconsiderando a intermitência enteroparasitária.

Outro fator que pode colaborar com possível prevalência subestimada é a automedicação, que favorece a resistência parasitária, sua proliferação no meio e a reinfecção de indivíduos.

\section{CONCLUSÃO}

A taxa de prevalência verificada em Limoeiro do Norte foi próxima da média mundial segundo a OMS, portanto há perspectiva de se reduzir a contaminação da população com medidas efetivas.

Os resultados obtidos corroboram com as variadas prevalências encontradas em regiões distintas, onde, neste caso, os parasitas mais frequentes foram Entamoeba coli, Endolimax nana e Giardia lamblia.

Adultos e adolescentes foram os indivíduos mais acometidos, assim como foi maior a prevalência identificada em UBSs situadas na zona rural, reforçando a idéia da heterogeneidade de ocorrência intramunicipal desta patologia, logo sendo importante planejamento estratégico, focado no rompimento do ciclo epidemiológico do parasita por meio de ampliação de serviços de saneamento básico, por exemplo.

De maneira sazonal, foi identificada diferença significativa entre os casos positivos no decorrer do biênio, com maior ocorrência no último semestre do período.

Por fim, mesmo com a alta prevalência identificada, não se pode descartar o fato de a mesma estar subestimada, devido a fatores de ordem metodológica do diagnóstico e a possível prática de automedicação por parte dos pacientes.

\section{AGRADECIMENTOS}

À CAPES pela bolsa de mestrado do primeiro autor, e ao SAAE de Limoeiro do Norte e à SMS pela disponibilização dos dados.

\section{REFERÊNCIAS BIBLIOGRÁFICAS}

1. ADDUM, F.M.; SERRA, C.G.; SESSA, K.S.; IZOTON, L.M.; SANTOS, T.B., Planejamento local, saúde ambiental e Estratégia Saúde da Família: uma análise do uso de ferramentas de gestão para a redução do risco de contaminação por enteroparasitoses no município de Venda Nova do Imigrante. Physis, v. 21, n. 3, p. 955 - 978, 2011.

2. ALMEIDA, A.; MOREIRA, M. J.; SOARES, S.; DELGADO, M. L.; FIGUEIREDO, J.; SILVA, E. CASTRO, A.; COSA, J. M. C. Presence of Cryptosporidium spp. and Giardia duodenalis in drinking water samples in the North of Portugal. The Korean Journal of Parasitology, v. 48, n.1, p. $43-48,2010$.

3. BARRETO, M.L.; GENSER, B.; STRINA, A.; TEIXEIRA, G.M.; ASSIS, A.M.D.; REGO, R.; TELES, C.A.; PRADO, M.; MATOS, S.M.A; SANTOS, DN; SANTOS, L.A.; CAIRNCROSS, S. Effect of the city- 
wide sanitation programme on reduction in rate of childhood diarrhea in northeast Brazil: assessment by two cohort studies. Lancet v. 370, n. 9599, p. $1622-1628,2007$.

4. BASSO, R.M.C.; SILVA-RIBEIRO, T.; SOLIGO, D.S.; RIBACKI, S.I.; CALLEGAR-I-JACQUES, S.M.; ZOPPAS, B.C.A. Evolução da prevalência de parasitoses intestinais em escolares em Caxias do Sul, RS. Revista da Sociedade Brasileira de Medicina Tropical, v. 41, n.3, p. 263 - 268, 2008.

5. BELLIN, M.; GRAZZIOTIN, N.A. Prevalência de parasitos intestinais no município de Sanandauva/RS, Newslab, v. 18, n. 104, p. 116 -122, 2011.

6. BELO, V.S. et al. Factors associated with intestinal parasitosis in a population of children and adolescents. RevistaPaulista de Pediatria, v. 30, n. 2, p. 195 - 201, 2012.

7. BORGES, W.F.; MARCIANO, F.M.; OLIVEIRA, H.B. Parasitos intestinais: elevada prevalência de Giardialamblia em pacientes atendidos pelo serviço público de saúde na região sudeste de Goiás, Brasil. Revista de Patologia Tropica, v. 40, n.2, p. 149 - 157, 2011.

8. CIMERMAN, B.; CIMERMAN, S. Parasitologia humana e seus fundamentos gerais. São Paulo: Atheneu, 2008.

9. COSTA, A.C.N.; BORGES, B. C.; COSTA, A. V.; RAMOS, M. F.; GOMES, J. M.; GOMES, J. M; BUENO, H.; FARIA, T. A. Levantamento de acometidos por enteroparasitoses de acordo com a idade e sexo e sua relação com o meio onde está inserido o PSF Prado na cidade de Paracatu - MG. Revista de Patologia Tropical, v. 41, n. 2, p. 203 - 224, 2012.

10. COURA, C.F.; WILLCOX, H. P. F.; TAVARES, A. M.; PAIVA, D. D.; FERNANDES, O. RADA, E. L. J. C.; PereZ, E. P.; BORgeS, L. C. L.; HIDAlgo, M. E. C.; NOGUeIRA, M. L. C. Aspectos epidemiológico, sociais e sanitários de uma área do Rio Negro, Estado do Amazonas, com especial referência às parasitoses intestinais e à infecção chagásica. Caderno de Saúde Pública, v. 10, supl. 2, p. $331-334,1994$.

11. DIAS, D.G. Prevalência estacional de enteroparasitoses em uma população de zero a quatorze anos no bairro COHAB Tablada, Pelotas, RS. Pelotas, 2005. Dissertação de Mestrado - Programa de Pós-Graduação em Parasitologia - Universidade Federal de Pelotas, 2005.

12. FERNANDES, S.; BEORLEGUI, M.; BRITO, M. J.; ROCHA, G. Protocolo de parasitoses intestinais. Acta Pediátrica Portuguesa, v. 43, n. 1, 2011.

13. FERREIRA, H. LALA, E. R. P.; MONTEIRO, M. C. RAIMUNDO, M. L. Estudo epidemiológico localizado da frequência e fatores de risco para as enteroparasitoses e sua relação com o estado nutricional de crianças em idade pré-escolar. Ciências Biológicas e Saúde, v. 12, n. 4, p. $33-40,2006$.

14. FOnSECA, E.O.L.; TeIXeIRA, M. G.; BARRETO, M. L.; CARMO, E. H.; COSTA, M. C. N. Prevalência e fatores associados às geo-helmintoses em crianças residentes em municípios com baixo IDH no Norte e Nordeste. Caderno de Saúde Pública, v. 26, n. 1, p. 143 - 152, 2010.

15. FOnTBOnNE, A.; FREeSE-DE-CARVAlHO. E.; ACIOLI, M. D.; SÁ, G. A.; CESSE, E. A. P. Fatores de risco para o poliparasitismo intestinal em uma comunidade indígena em Pernambuco. Caderno de Saúde Pública, v. 17, n. 2, p. 367 - 373, 2001.

16. FUNCEME. Gráfico de chuvas dos postos pluviométricos. Disponível em: < http://www.funceme.br/index.php/areas/tempo/grafico-de-chuvas-dos-postospluviometricos >. Consultado em 21 de dezembro de 2013, 2013. 
17. FURTADO, L.F.V.; MELO, A.C.F.L. Prevalência e aspectos epidemiológicos na população geronte de Parnaíba, Estado do Piauí. Revista Sociedade Brasileira de Medicina Tropical, v. 44, n. 4, p. $515-523,2011$.

18. GOMES, R; NASCIMENTO, E.F. ARAÚJO, F.C. Por que os homens buscam menos os serviços de saúde do que as mulheres? As explicações de homens com baixa escolaridade e homens com ensino superior. Caderno de Saúde Pública, v.23, n. 3, p. 565 - 574, 2007.

19. HOFFMANN, W. A.; PONS, J. A.; JANER, J. L..Sedimentation concentration method in schistosome. Porto Rico, 1934.

20. IBGE. Censo 2010. Disponível em: http://www.censo2010.ibge.gov,br/ . Consultado em: 12 de agosto de 2013, 2010.

21. LIMOEIRO DO NORTE. Plano Municipal de Saneamento Básico, 2009.

22. LODO, M.; OLIVEIRA, C. G. B.; FONSECA, A. L. A.; CAUPUTTO, L. Z.; PACKER, M. L. T.; VALENTI, V. E.; FONSECA, F. L. A. Prevalência de enteroparasitas em município do interior paulista. Revista Brasileira de Crescimento e Desenvolvimento Humano, v. 20, n. 3, p. 769 - 777, 2010.

23. LOPES, A.C.M; BITENCOURT, F.C.; MELO, C.M.; MADI, R.R; ANDRADE, R.M.S.; BRITO, A.M.G. Geohelmintíase: prevalência amostral em Aracaju (SE) entre 2007 a 2010. ScireSalutis, v.3, n. 1, p. $28-36,2013$.

24. LUDWING, K,M.; FREI, F. ALVARES FILHO, F.; RIBEIRO-PAES, J.T. Correlação entrecondições de saneamento básico e parasitoses intestinais na população de Assis, Estado de São Paulo. Revista da Sociedade Brasileira de Medicina Tropical, v. 32, n. 5, 1999.

25. MAGALHÃES, V.M.; CARVALHO, A.G.; FREITAS, F.I.S. Inquérito parasitológico em manipuladores de alimentos em João Pessoa, PB, Brasil. Revista de Patologia Tropical, v. 39, n. 4 , p. $335-342,2010$.

26. MATI, V.L.T.; PINTO, J.H. MELO, A.L. Levantamento de parasitoses intestinais nas áreas urbana e rural de Itambé do Mato Dentro, Minas Gerais, Brasil. Revista de Patologia Tropical, v. 40 , n. 1. p. $92-100,2011$.

27. MATOS, M.A.; CRUZ, Z.V. Prevalência de parasitoses intestinais no município de Ibiassucê Bahia. Revista Educação, Meio Ambiente e Saúde, v. 5, n. 1, p. 64 - 71, 2012.

28. MENEZES, R.A.D.; GOMES, M.S.M.; BARBOSA, F.H.F.; BRITO, G.C.M.; PROJETTI JÚNIOR, A. A.; COUTO, A.A.R.D. Enteroparasitoses em pacientes atendidos no laboratório de uma unidade de saúde de Macapá, Amapá, 2011 - 2012. Revista de Biologia e Ciências da Terra, v. 13, n. 1, p. $191-198,2013$.

29. OLIVEIRA, C.H.B. Frequência de enteroparasitoses em usuários do serviço público de saúde na mesorregião do Oeste Potiguar. Natal, 2013. Dissertação de Mestrado - Saúde Coletiva Universidade Estadual do Rio Grande do Norte, 2013.

30. OLIVEIRA, U.D.; CHIUCHETTA, S.J.R. Ocorrência de enteroparasitoses na população do município de Goioerê - PR. Uniciências, v. 14, n. 2, p. 155 - 158, 2010.

31. REY, L. Bases da parasitologia médica. Guanabara Koogan. Rio de Janeiro, 2002. p. 410.

32. ROCHA, T.J.M.; BRAZ, J.C.; CALHEIROS, C.M.L.P arasitismo intestinal em uma comunidade carente no município de Barra de Santo Antônio, Estado de Alagoas. Revista Eletrônica de Farmácia, v.2, n. 3, p. $128-136,2010$. 
33. RODRIGUES, J.A.; CARNEIRO, W.S.; ATHAYDE, A.C.R. Infecções por helmintos gastrointestinais: perfil de crianças em escolas públicas e privadas do Sertão Paraibano. Newslab, v. 118, p. 128 - 136, 2013.

34. SANTOS, A.S.; MERLINI, L.S. Prevalência de enteroparasitoses na população do município de Maria Helena, Paraná. Ciência Saúde Coletiva, v. 14, n. 3, 2010.

35. SANTOS, L.P.; SANTOS, F.L.N.; SOARES, N.M. Prevalência de parasitoses intestinais em pacientes atendidos no Hospital Universitário Professor Edgar Santos, Salvador - Bahia. Revista de Patologia Tropical, v.36, n. 3, p. 237 - 246, 2007.

36. SILVA, F.S. Frequência de parasitos intestinais no município de Chapadinha, Maranhão, Brasil. Revista de Patologia Tropical, v. 39, n. 1, p. 63 - 68, 2010.

37. SILVA JUNIOR, J.R. Parasitoses intestinais e saneamento ambiental: estudo da situação dos bairros Bela Vista e Ulisses Guimarães em Teixeira de Freitas - BA. Aracruz, 2010. Dissertação de Mestrado - Mestrado Profissional em Tecnologias Ambientais. Faculdade de Aracruz, 2010.

38. SILVA, E.F.; , E. B.; ALMEIDA, K. S.; SOUSA, J. J. N. FREITAS, F. L. C. Enteroparasitoses em crianças de áreas rurais do município de Coari, Amazonas, Brasil. Revista de Patologia Tropical, v. 38, n. 1, p. $35-43,2009$.

39. SOUZA, V.F. LUCCA, G. ANIBAL, F.F. Mapeamento epidemiológico das parasitoses intestinais em seis bairros do município de Araraquara/SP. Revista Uniara, n. 19, p. 31 - 40, 2006.

40. TOLEDO, M.J.O; PAULUDETTO, A.W.; MOURA, F.T.; NASCIMENTO, E.S.; CHAVES, M.; ARAÚJO, S.M.; MOTA, L.T.Avaliação de atividades de controle para enteroparasitos em uma aldeia Kaingángdo, Paraná. Revista de Saúde Pública, v. 43, n. 6, p. 981 - 990, 2009. 